\title{
Characteristics and Treatment Outcomes of "Transfer-Out" Pulmonary Tuberculosis Patients in Gondar, Ethiopia
}

\author{
Tadesse Belayneh, ${ }^{1}$ Afework Kassu, ${ }^{2}$ Desalgne Tigabu, ${ }^{3}$ Gashaw Asmare, ${ }^{4}$ \\ Sofanit Tilaye, ${ }^{4}$ and Eveline Klinkenberg ${ }^{5,6}$ \\ ${ }^{1}$ Department of Medical Anesthesiology, CMHS, University of Gondar, P.O. Box 196, Gondar, Ethiopia \\ ${ }^{2}$ Department of Microbiology Immunology and Parasitology, CMHS, University of Gondar, P.O. Box 196, Gondar, Ethiopia \\ ${ }^{3}$ Institute of Public Health, CMHS, University of Gondar, P.O. Box 196, Gondar, Ethiopia \\ ${ }^{4}$ University of Gondar Hospital, P.O. Box 196, Gondar, Ethiopia \\ ${ }^{5}$ KNCV Tuberculosis Foundation, The Hague, Netherlands \\ ${ }^{6}$ Department of Global Health, Academic Medical Center, Amsterdam Institute for Global Health and Development, \\ University of Amsterdam, Amsterdam, Netherlands
}

Correspondence should be addressed to Tadesse Belayneh; tadbel20@gmail.com

Received 10 February 2016; Revised 18 April 2016; Accepted 21 April 2016

Academic Editor: David C. Perlman

Copyright (C) 2016 Tadesse Belayneh et al. This is an open access article distributed under the Creative Commons Attribution License, which permits unrestricted use, distribution, and reproduction in any medium, provided the original work is properly cited.

Background. During tuberculosis treatment, patients may transfer to continue treatment at another health facility. To ensure adherence until treatment completion, keeping track of patients is paramount. This study aimed to investigate treatment outcomes of patients who transferred out from the University of Gondar Hospital. Methods. This was a retrospective cohort evaluation of patients registered from 2009 to 2013. Treatment outcomes were collected from the TB registers of receiving units using a standardized data capture format. Results. During the study period 3,707 patients initiated treatment and $47.5 \%(1,760)$ transferred out. The study evaluated the outcome of $26 \%(457 / 1,760)$ patients, of whom 403 (88\%) arrived in the receiving units. Overall, $79 \%$ were successfully treated and $13.8 \%$ transferred out for a second time. For all transferred-out cases, treatment outcomes were not reported to the referring unit. Conclusion and Recommendation. About half of the patients were transferred out to complete treatment elsewhere. Although successful treatment outcome was obtained in $79 \%$ of patients, these results were not fed back to the referring unit. Implementing a clear mechanism to communicate the arrival of and treatment outcome for transfer-out patients and appropriate patient education on treatment unit selection before treatment and during transfer-out are recommended.

\section{Introduction}

In Ethiopia, tuberculosis (TB) remains a major public health problem [1]. According to the Global TB Report 2015, Ethiopia is one of the 30 high TB burden countries and one of the 14 that have a high burden of TB, TH-HIV, and MDRTB [2]. The directly observed treatment short course (DOTS) strategy has been implemented in the country since 1994 to control the TB epidemic [3]. Despite 100\% geographical and $92 \%$ health facility coverage of the DOTS programme [3], noncompliance, low case detection, a high transfer-out rate, and deficient recording and reporting systems are still a challenge [4].
Globally, all national TB control programmes experience the challenges of intracountry movement of patients from one treatment center to another [2]. A transfer-out patient is a patient who has been transferred out at any time during treatment to continue treatment at another facility [5]. The proportion of transferred-out patients varies from one country to another. Reports indicate that in Sub-Saharan Africa it can range from $1 \%$ to $26 \%[6,7]$. In Ethiopia figures from $31 \%$ to $66 \%$ have previously been reported [8-10].

Treatment outcome is a key indicator in TB control and serves as a proxy of quality of TB treatment in a health care system [11]. A study in the UoGH showed that between 2003 and 2008, 42\% of TB patients were transferred out 
to continue their treatment at nearby health units [9]. The Ethiopian Clinical and Programmatic Management of TB, TB/HIV and Leprosy guidelines [2] indicate that the outcome for transferred-out patients should be reported to the national surveillance system by the transferring unit, as this unit notified and initiated treatment for this patient. Monitoring treatment outcomes is essential in order to evaluate the effectiveness of the DOTS program [12]. This would make it possible to recognize and amend system failures before the incidence and proportion of resistant isolates rise [11]. However, there are limited studies conducted to assess the treatment outcome of PTB patients who are transferred out. Moreover, in view of the large number of transferred out patients in $\mathrm{UoGH}$, it is important to know what their final treatment outcomes are and whether they are available at the transferring facility for them to be captured in the TB surveillance system. Therefore, this study was conducted to assess the TB treatment outcomes of transferred-out PTB patients who were registered and started treatment at UoGH.

\section{Methods}

2.1. Study Area. UoGH is a tertiary hospital serving the population of Gondar town and the remote hilly areas of northwest Ethiopia. The hospital has a DOTS clinic that has been operational since 2000 [13]. Around $740 \mathrm{~TB}$ patients are diagnosed and treated according to the national guidelines annually. The primary aim of the study was to evaluate treatment outcomes of PTB patients who had been transferred out from UoGH and who had arrived at their receiving centers. For logistic and resource reasons, only centers which were within a $180 \mathrm{~km}$ radius of Gondar town were included; these were fifteen centers.

2.2. Study Design and Data Collection. A retrospective analysis of the profile of transferred-out PTB patients registered at DOTS clinic of UoGH in the years 2009 to 2013 was conducted. Data were collected from March to June 2014. The registration documents reviewed contained basic information, such as age, sex, and sputum status, type of TB by history, HIV status, and phase of treatment at the time of transfer. Patients whose names, father's names, age, and sex matched between the registers at the UoGH and the receiving units and were classified as "transfers-in" in the receiving units were defined as "arrivals." Patients whose names did not appear in the receiving units were defined as "nonarrivals." For "arrivals," treatment outcome was collected from the TB treatment registers of the receiving centers.

2.3. Definitions. The treatment outcome was categorized according to National TB and Leprosy Control Program (NTLCP) guidelines [13]. These categories were being cured (finished treatment with negative bacteriology result at the end of treatment), completed treatment (finished treatment, but without bacteriology result at the end of treatment for those who are smear-positive), failure (remaining smearpositive at five months despite correct intake of medication), default (patients who interrupted their treatment for two consecutive months or more after registration), death (patients who died from any cause during the course of treatment), transfer-out (patients whose treatment results are unknown due to transfer to another health facility), and successful treatment (a patient who was cured or completed treatment).

2.4. Statistical Analyses and Ethical Considerations. Data were entered, cleaned, and analyzed using the statistical package SPSS for Windows, version 20. Inconsistencies in data entry were randomly checked by reentering $10 \%$ of the raw data. Frequencies and percentages were used to describe patient characteristics and treatment outcomes. The study was approved by the Institutional Review Board of the University of Gondar. In addition, official permission was secured from the Regional Health Bureau of Amhara region as well as the involved treatment units.

\section{Results}

3.1. Characteristics of Transferred-Out Patients. During the study period 3,707 PTB patients were registered and initiated the treatment at UoGH DOTS clinic. Among these, $47.5 \%$ $(1,760 / 3,707)$ were transferred out to complete their treatment in different treatment units after his/her transfer request.

Of these patients, $25.9 \%(457 / 1,760)$ were transferred to the fifteen selected units and their outcome was evaluated. Most of these transferred-out patients (see Table 1) were smear-negative (76.4\%), male (59.3\%), and new (91.9\%) and in the age group of $19-34$ years $(40.0 \%)$. All the transferredout patients were offered Provider Initiated HIV Testing and Counseling (PITC) at UoGH and accepted to be tested. The TB-HIV coinfection rate was $37.9 \%$ (173/457) and 44 of those patients $(27.1 \%)$ were on cotrimoxazole prophylaxis and $54(33.3 \%)$ on antiretroviral therapy at the time of transfer. About two-thirds of the patients (309/457, 68\%) were transferred out during the intensive phase. The median time between treatment initiation and transfer-out was 46 days (range 9 to 140 days).

In the $\mathrm{TB}$ registers of the receiving units, only $88.2 \%$ (403/457) patients were traced and classified as "arrivals." The remaining $54(11.8 \%)$ were classified as "nonarrivals." Of the "nonarrivals," 9 (16.6\%) were pulmonary smear-positive and $17(31.5 \%)$ were HIV positive (Table 1$)$.

\subsection{Treatment Outcomes of Transferred-Out PTB Patients.} TB treatment outcomes were analyzed for the 403 "arriving" patients only (Table 2). More than three-quarters, $78.8 \%$ $(318 / 403)$, of patients had a successful treatment outcome (18.8\% cured and $60.0 \%$ completed treatment) while 85 (21.1\%) had an unsuccessful outcome (1.7\% defaulted, $4.9 \%$ died, $0.5 \%$ failed, and $13.8 \%$ transferred out). For all 457 transferred-out patients, feedback on treatment outcomes was not reported to UoGH by the receiving units.

\section{Discussion}

Monitoring treatment outcomes is essential in order to evaluate the effectiveness of the DOTS program [12]. Many patients are diagnosed and initiate treatment at large hospitals. To 
TABLE 1: Characteristics of transferred-out PTB patients at the University of Gondar Hospital and those who arrived and did not arrive at selected receiving units, respectively, Ethiopia, 2009-2013.

\begin{tabular}{|c|c|c|c|}
\hline Characteristics & $\begin{array}{c}\text { Transfer-out }(N=457) \\
\text { number }(\%)\end{array}$ & $\begin{array}{l}\text { Arriving }(N=403) \\
\text { number }(\%)\end{array}$ & $\begin{array}{c}\text { Nonarriving }(N=54) \\
\text { number }(\%)\end{array}$ \\
\hline \multicolumn{4}{|l|}{$\operatorname{Sex}$} \\
\hline Male & $271(59.3)$ & $244(90.0)$ & $27(10.0)$ \\
\hline Female & $186(40.7)$ & $159(85.5)$ & $27(14.5)$ \\
\hline \multicolumn{4}{|l|}{ Age (years) } \\
\hline$\leq 18$ & $137(30.0)$ & $111(81.1)$ & $26(18.9)$ \\
\hline $19-34$ & $183(40.0)$ & $168(91.8)$ & $15(8.2)$ \\
\hline $35-54$ & $106(23.2)$ & $98(92.5)$ & $8(7.5)$ \\
\hline$\geq 55$ & $31(6.8)$ & $26(83.9)$ & $5(16.1)$ \\
\hline \multicolumn{4}{|c|}{ Sputum status (pulmonary TB type) } \\
\hline Smear-positive & $108(23.6)$ & $99(91.7)$ & $9(8.3)$ \\
\hline Smear-negative & $349(76.4)$ & $304(87.1)$ & 45 (12.9) \\
\hline \multicolumn{4}{|l|}{ Type of TB by history } \\
\hline New & $420(91.9)$ & $368(87.6)$ & $52(12.4)$ \\
\hline Relapse & $27(5.9)$ & $26(96.3)$ & $1(3.7)$ \\
\hline Return after default & $4(0.9)$ & $4(100.0)$ & $0(0.0)$ \\
\hline Failure & $6(1.3)$ & $5(83.3)$ & $1(16.7)$ \\
\hline \multicolumn{4}{|l|}{ HIV status } \\
\hline Negative & $284(62.1)$ & $247(86.9)$ & $37(13.1)$ \\
\hline Positive & $173(37.9)$ & $156(90.2)$ & $17(9.8)$ \\
\hline \multicolumn{4}{|c|}{$\begin{array}{l}\text { Duration in which the patient transferred } \\
\text { out after initiation of treatment }\end{array}$} \\
\hline$<4$ weeks & 85 (18.6) & $79(92.9)$ & $6(7.1)$ \\
\hline $4-12$ weeks & $365(79.9)$ & $317(86.8)$ & $48(13.2)$ \\
\hline$>12$ weeks & $7(1.5)$ & $7(100.0)$ & $0(0.0)$ \\
\hline
\end{tabular}

enhance treatment adherence, these hospitals often transfer out patients to a clinic closer to the patients' homes, which has obvious benefits for patients and their families [14, 15]. Different studies have shown that receiving care at only one health facility improves patient compliance thereby reducing the risk of default $[9,10]$. Moreover, receiving care close to home is also a key factor for compliance, as when people travel far for daily/weekly DOT they are more likely to default $[10,16]$. The current study showed that nearly half of the patients were transferred out after being started on treatment. Other similar studies in the same region reported proportions in the $31.4 \%-$ $42.0 \%$ range $[8,9]$. The proportion of transferred-out patients in the current study was slightly higher than observed at a previous study in UoGH reporting $42 \%$ of patients were transferred out [9] and another study in the region that reported $31.4 \%$ [8]. The current observed higher proportion could be due to the establishment and expansion of treatment units, securing supplies of $\mathrm{TB}$ medicines in several health facilities and improving management in the TB program. These all lead patients to seek continued TB treatment in the nearby institutions. The result of current study is lower compared to a study conducted at one hospital in the same region (Felege Hiwot Hospital in Bahir Dar) in which 65.6\% of patients were transferred out [10].
No patient should be lost when transferred out between treatment units. However, in the current study, $12 \%$ of patients were classified as "nonarrivals" as their names could not be identified in the register of the receiving unit. This proportion of $12 \%$ was higher compared to studies conducted in Laos and Afghanistan in which $4 \%$ and $10 \%$ of patients did not arrive and continue treatment at receiving units correspondingly $[14,17]$. For these "nonarrivals" it could not be ensured whether they had continued their treatment. If not they continue to spread TB, especially when they are pulmonary smear-positive. In addition, such treatment interruption could favor the development of drug resistant TB. The possible reasons for these "nonarrivals" might be self-referral to another preferred facility before reaching the destined receiving unit or they may have died or discontinued treatment. This could be linked to patients not following instructions from the health care providers or providers not communicating clearly enough with patients or a combination of both. This needs further investigation.

A high transfer-out rate often results in a lower treatment success rate as for those for whom the result is not reported back the result is listed as not-evaluated.

This could jeopardize achieving the set TB control targets. The Ethiopian National Strategic Plan (2010-2015) has set a 
TABLE 2: Treatment outcomes of transferred PTB patients who arrived at their receiving facility in northwest Ethiopia, 2009-2013 ( $N=403)$.

\begin{tabular}{|c|c|c|c|c|c|c|}
\hline \multirow{2}{*}{ Characteristics } & \multicolumn{6}{|c|}{ Treatment outcomes $n(\%)$} \\
\hline & Cured & Treatment completed & Default & Failure & Death & Transfer-out \\
\hline \multicolumn{7}{|l|}{ Age (years) } \\
\hline$\leq 18$ & $17(22.4)$ & $72(29.8)$ & $0(0)$ & $0(0)$ & $6(30.0)$ & $16(28.6)$ \\
\hline $19-34$ & $29(38.2)$ & $96(39.7)$ & $6(85.7)$ & $2(100.0)$ & $8(40.0)$ & $27(48.2)$ \\
\hline $35-54$ & $24(31.6)$ & $57(23.6)$ & $1(14.3)$ & $0(0)$ & $4(20.0)$ & $12(21.4)$ \\
\hline$\geq 55$ & $6(7.9)$ & $17(7.0)$ & $0(0)$ & $0(0)$ & $2(10.0)$ & $1(1.8)$ \\
\hline \multicolumn{7}{|l|}{$\operatorname{Sex}$} \\
\hline Male (=244) & $43(17.6)$ & $145(59.4)$ & $4(1.6)$ & $1(0.4)$ & $14(5.7)$ & $37(15.2)$ \\
\hline Female $(=159)$ & $33(20.7)$ & $97(61.0)$ & $3(1.9)$ & $1(0.6)$ & $6(3.8)$ & $19(11.9)$ \\
\hline \multicolumn{7}{|c|}{ Sputum status (pulmonary type of TB) } \\
\hline Smear-positive & $42(42.4)$ & $35(35.3)$ & $2(2.0)$ & $0(0)$ & $4(4.0)$ & $16(16.2)$ \\
\hline Smear-negative & $34(11.2)$ & $207(60.1)$ & $5(1.6)$ & $2(0.6)$ & $16(5.3)$ & $40(13.1)$ \\
\hline \multicolumn{7}{|c|}{ Categories of TB at start } \\
\hline New & $66(17.9)$ & $223(60.6)$ & $6(1.6)$ & $2(0.5)$ & $19(5.2)$ & $52(14.1)$ \\
\hline Relapse & $8(30.8)$ & $14(53.8)$ & $0(0)$ & $0(0)$ & $1(3.8)$ & $3(11.5)$ \\
\hline Default & $1(25.0)$ & $2(50.0)$ & $0(0)$ & $0(0)$ & $0(0)$ & $1(25.0)$ \\
\hline Failure & $1(20.0)$ & $3(60.0)$ & $1(20.0)$ & $0(0)$ & $0(0)$ & $0(0)$ \\
\hline \multicolumn{7}{|l|}{ HIV status } \\
\hline Positive (=155) & $23(14.8)$ & $96(61.9)$ & $1(0.6)$ & $1(0.6)$ & $11(7.1)$ & $23(14.8)$ \\
\hline Negative (=248) & $53(21.4)$ & $146(58.8)$ & $6(2.4)$ & $1(0.4)$ & $9(3.6)$ & $33(13.3)$ \\
\hline \multicolumn{7}{|c|}{$\begin{array}{l}\text { Time period between transfer-out and } \\
\text { transfer-in ( } 363 \text { ) }\end{array}$} \\
\hline Same to 2nd day & $31(19.3)$ & $91(56.8)$ & $3(1.8)$ & $1(0.6)$ & $13(8.1)$ & $21(13.1)$ \\
\hline 3rd day & $35(19.2)$ & $110(60.4)$ & $4(2.2)$ & $1(0.5)$ & $5(2.7)$ & $27(14.8)$ \\
\hline$\geq 4$ th day & $5(23.8)$ & $14(66.6)$ & $0(0)$ & $0(0)$ & $1(4.8)$ & $1(4.8)$ \\
\hline \multicolumn{7}{|c|}{$\begin{array}{l}\text { Phase of TB treatment at time of } \\
\text { transfer-out }\end{array}$} \\
\hline Intensive & $59(21.1)$ & $163(58.2)$ & $5(1.9)$ & $1(0.3)$ & $14(5.0)$ & $38(13.5)$ \\
\hline Continuation & $17(13.8)$ & $79(64.2)$ & $2(1.6)$ & $1(0.8)$ & $6(4.8)$ & 18 (14.6) \\
\hline Overall & 76 (18.9\%) & $242(60.0 \%)$ & $7(1.7 \%)$ & $2(0.5 \%)$ & $20(4.9 \%)$ & $56(13.8 \%)$ \\
\hline
\end{tabular}

treatment success target of $90 \%$ [1], equal to the global set target by the WHO [18]. In this study, the overall treatment success rate for PTB patients who were transferred out was $79 \%$ which is lower than both the national and the global target. Currently, Ethiopia reports a treatment success rate of $91 \%$ for new cases registered in 2012, while for previously treated cases this is $43 \%$ [1]. The study result of $79 \%$ treatment success is below this and also lower than previously reported treatment success rates in northwest Ethiopia ranging from $86 \%$ to $95 \%$ [19-21] and than Amhara region which reported a treatment success rate of $87.5 \%$ in 2014 [22].

If TB control activities function, all patients who transfer out to other units should be recorded as transfers-in and the treatment outcome results communicated back to the referring units via feedback reports and by telephone [15, 23]. In the current study, none of the transferred-in TB patients had an outcome communicated to their referral institution. This suggests that TB reporting systems might not function optimally with regard to communication on treatment outcome of transferred patients. Reasons for this were unclear but it could be due to unawareness of the need for the procedure, negligence, expecting the other to report, and/or lack of communication modalities [24]. Lack of clear guidance and communication between referring and accepting DOTS clinics requires evaluation and immediate attention. The primary DOTS clinic should have collected treatment outcome data from the accepting clinics. Efficient counseling and follow-up of transferred-out patients are mandatory to avoid poor treatment outcomes. Developing clear guidance on how to trace and report transferred-out cases is essential to avoid spillover of TB and MDR-TB [24]. Telecommunication facilities are an area in need of improvement as many of the DOT clinics lack this. Also, the reporting of transferred patients may cause double counting of patients during routine quarterly reporting to central units if both the referring and receiving facility are notifying the patient. This should be looked into. 


\section{Limitations of the Study}

The current study had several limitations: Firstly, it is specific to one setting which may make it difficult to extrapolate the findings to other settings. Secondly, due to the inherent features of a retrospective study design missing data/incomplete records may have influenced the results. Thirdly, the study unfortunately did not include a control group of patients that were not transferred out for comparison purposes. Fourthly, the linking between facility records was done using name matching and not with a unique identifier. Also, TB treatment outcomes were not available for the "nonarrivals" and their exclusion might have biased the treatment success rate. Despite the above limitations, the study result provided important insights into TB control for policy makers and TB program managers and identified the need to strengthen communication for transferred-out cases to ensure the surveillance data accurately reflect the number of patients on treatment as well as treatment outcomes of all $\mathrm{TB}$ cases.

\section{Conclusion and Recommendation}

In conclusion, at UoGH about half of TB patients were transferred out to complete treatment elsewhere while $14 \%$ of them were transferred out for a second time. Successful treatment outcome was obtained in $79 \%$ of transferred-out patients. However these results are not fed back to the referring facilities that should formally report the outcomes for these cases. This results in underreporting of successful treatment outcomes. A clear mechanism should be implemented to communicate the arrival of and treatment outcomes for transfer-out patients to ensure all patient information is up to date for adequate monitoring of TB control activities. Appropriate patient education on treatment unit selection before treatment and during transfer-out is also recommended.

\section{Abbreviations}

DOTS: Directly observed treatment short course

HIV: Human Immunodeficiency Virus

TB: Tuberculosis

UoGH: University of Gondar Hospital

WHO: World Health Organization.

\section{Ethical Approval}

Retrospective data were collected from TB registers after approval and permission from UoG Ethics Review Board.

\section{Consent}

No individual patient consent was sought as patients were not approached.

\section{Disclosure}

The contents are the responsibility of the authors and do not necessarily reflect the views of USAID or the United States Government.

\section{Competing Interests}

No competing interests exist regarding the publication of this paper.

\section{Authors' Contributions}

Tadesse Belayneh conceived the idea, designed the research, analyzed and interpreted the data, and drafted and critically revised the paper. Afework Kassu, Desalgne Tigabu, and Eveline Klinkenberg advised on the protocol and participated in statistical analysis and drafting and critical reviewing of the paper for important intellectual content. Gashaw Asmare and Sofanit Tilaye handled supervision of overall work and revised the paper critically for important intellectual content. All authors read and approved the final paper.

\section{Acknowledgments}

This study was conducted under the Ethiopia Operational Research Capacity Building Initiative of TRAC/FMOH supported by USAID/TB CARE I project. The authors thank the Global Health Bureau, Office of Health, Infectious Disease and Nutrition (HIDN) of the US Agency for International Development, which financially supported this study through the TB CARE I mechanism under the terms of Agreement no. AID-OAA-A-10-00020. This study was made possible by the generous support of the American people through the United States Agency for International Development (USAID). The authors also sincerely thank Dr. Riitta Dlodlo from the International Union against Tuberculosis and Lung Disease, for her constructive comments on the paper. The authors also sincerely thank the data collectors, health facility managers, and TB focal persons for their cooperation.

\section{References}

[1] World Health Organization, Global Tuberculosis Report 2014, WHO/HTM/TB/2014.08, World Health Organization, Geneva, Switzerland, 2014.

[2] World Health Organization, Global Tuberculosis Report 2015, World Health Organization, Geneva, Switzerland, 20th edition, 2015, http://www.who.int/tb/publications/global_report/en/.

[3] Ministry of Health of Ethiopia, Tuberculosis, Leprosy and TB/HIV Prevention and Control Programme Manual, $\mathrm{MOH}$, Addis Ababa, Ethiopia, 2011.

[4] Federal Democratic Republic of Ethiopia, Health Sector Development Programme IV 2010/11-2014/15, 2010.

[5] World Health Organization, Definitions and Reporting Framework for Tuberculosis-2013 Revision, WHO/HTM/TB/2013.2, World Health Organization, Geneva, Switzerland, 2013.

[6] World Health Organization, Treatment of Tuberculosis: Guidelines for National Programmes, WHO/HTM/TB/2009.420, World Health Organization, Geneva, Switzerland, 4th edition, 2009.

[7] World Health Organization (WHO), Global Tuberculosis Control: Epidemiology, Strategy, Financing: WHO Report 2009 WHO/HTM/TB/2009.411, World Health Organization, Geneva, Switzerland, 2009. 
[8] A. Esmael, G. Tsegaye, M. Wubie, H. Abera, and M. Endris, "Treatment outcomes of TB patients in Debre Markos Referral Hospital, North West Ethiopia: a five year retrospective study," International Journal of Pharmaceutical Sciences and Research, vol. 5, no. 4, pp. 1500-1505, 2014.

[9] B. Tessema, A. Muche, A. Bekele, D. Reissig, F. Emmrich, and U. Sack, "Treatment outcome of tuberculosis patients at Gondar University Teaching Hospital, Northwest Ethiopia. A five-year retrospective study," BMC Public Health, vol. 9, article 371, 2009.

[10] F. Biadglegne, B. Anagaw, T. Debebe et al., "A retrospective study on the outcomes of tuberculosis treatment in Felege Hiwot Referral Hospital, Northwest Ethiopia," International Journal of Medicine and Medical Sciences, vol. 5, no. 2, pp. 85-91, 2013.

[11] T. Vasankari, P. Holmström, J. Ollgren, K. Liippo, M. Kokki, and P. Ruutu, "Risk factors for poor tuberculosis treatment outcome in Finland: a cohort study," BMC Public Health, vol. 7, article 291, 2007.

[12] J. Veen, M. Raviglione, H. L. Rieder et al., "Standardized tuberculosis treatment outcome monitoring in Europe. Recommendations of a Working Group of the World Health Organization (WHO) and the European Region of the International Union Against Tuberculosis and Lung Disease (IUATLD) for uniform reporting by cohort analysis of treatment outcome in tuberculosis patients," European Respiratory Journal, vol. 12, no. 2, pp. 505-510, 1998.

[13] Ministry of Health of Ethiopia, Tuberculosis, Leprosy and TB/HIV Prevention and Control Program Manual, $\mathrm{MOH}$, Addis Ababa, Ethiopia, 4th edition, 2012.

[14] T. Arnadottir, B. Phongosa, P. Chittamany, and H. Soukaseum, "Decentralizing tuberculosis treatment: follow-up of patients during the transitional period," International Journal of Tuberculosis and Lung Disease, vol. 6, no. 7, pp. 609-614, 2002.

[15] S. Meijnen, M. M. Weismuller, N. J. M. Claessens, J. H. Kwanjana, F. M. Salaniponi, and A. D. Harries, "Outcome of patients with tuberculosis who transfer between reporting units in Malawi," International Journal of Tuberculosis and Lung Disease, vol. 6, no. 8, pp. 666-671, 2002.

[16] B. Getahun, G. Ameni, S. Biadgilign, and G. Medhin, "Mortality and associated risk factors in a cohort of tuberculosis patients treated under DOTS programme in Addis Ababa, Ethiopia," BMC Infectious Diseases, vol. 11, pp. 127-134, 2011.

[17] M. Zhuben, F. M. Delawer, A. H. Andar, F. Salimi, and J. Ngamvithayapong-Yanai, "High tuberculosis treatment success in Kabul, Afghanistan despite high patient transfers out," Eastern Mediterranean Health Journal, vol. 19, no. 8, pp. 694697, 2013.

[18] World Health Organization, Global Tuberculosis Report 2012, World Health Organization, Geneva, Switzerland, 2012.

[19] M. G. Beza, T. W. Moges, D. T. Mulat, S. G. Yenework, M. B. Sisay, and S. B. Tefera, "A five years Tuberculosis treatment outcome at kolla diba health center, dembia district, northwest ethiopia: a retrospective cross-sectional analysis," Journal of Infectious Diseases and Therapy, vol. 1, article 101, 2013.

[20] S. Tadesse and T. Tadesse, "Treatment success rate of tuberculosis patients in Dabat, northwest Ethiopia," Health, vol. 6, no. 5, pp. 306-310, 2014.

[21] M. Endris, F. Moges, Y. Belyhun, E. Woldehana, A. Esmael, and C. Unakal, "Treatment outcome of tuberculosis patients at Enfraz Health Center, Northwest Ethiopia: a five-year retrospective study," Tuberculosis Research and Treatment, vol. 2014, Article ID 726193, 7 pages, 2014.
[22] Amhara Regional State Health Bureau, Tenth National TB Research Conference, Adama Town, October 2014, http://www .etharc.org/resources/download/finish.

[23] Federal Ministry of Health, "Overview of national TB control implementation status," in Proceedings of the Sixth National TB Research Workshop, Gondar University, June 2011.

[24] I. Shabbir, R. Iqbal, M. Anwar, E. Qadeer, and N. Ahmed, "An improved record system for tracing outcome of 'transferred-out' DOTS patients," Eastern Mediterranean Health Journal, vol. 17, no. 2, pp. 88-92, 2011. 


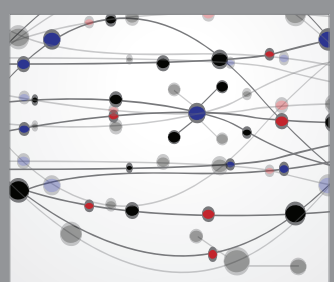

The Scientific World Journal
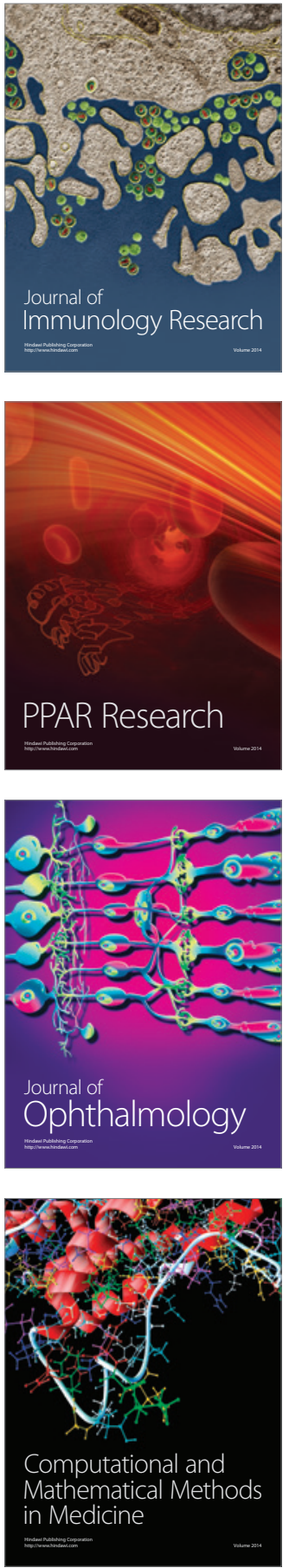

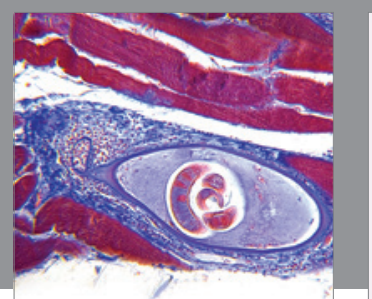

Gastroenterology Research and Practice

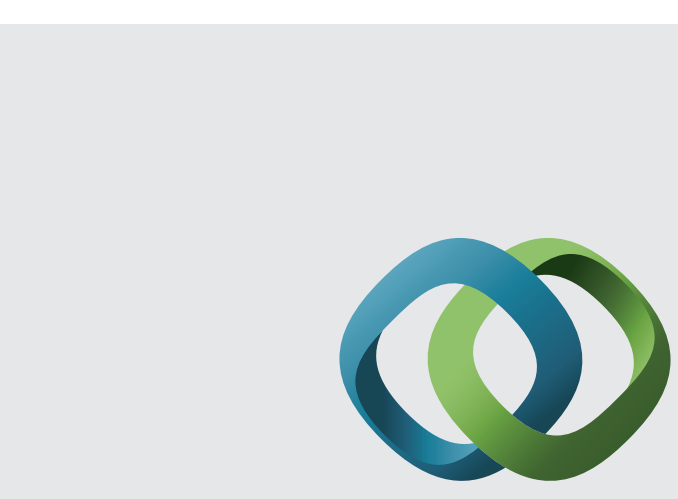

\section{Hindawi}

Submit your manuscripts at

http://www.hindawi.com
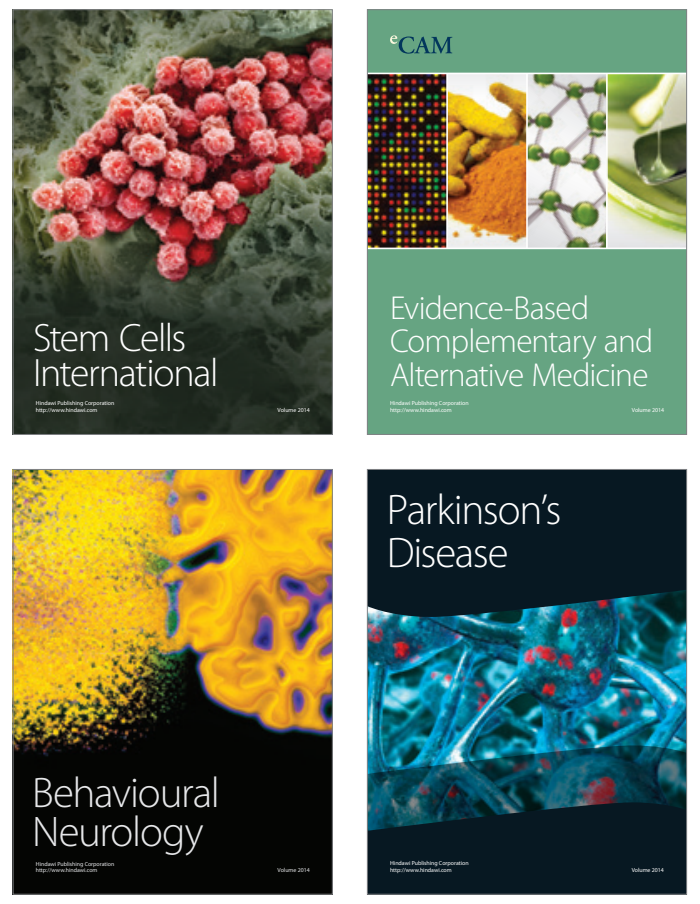
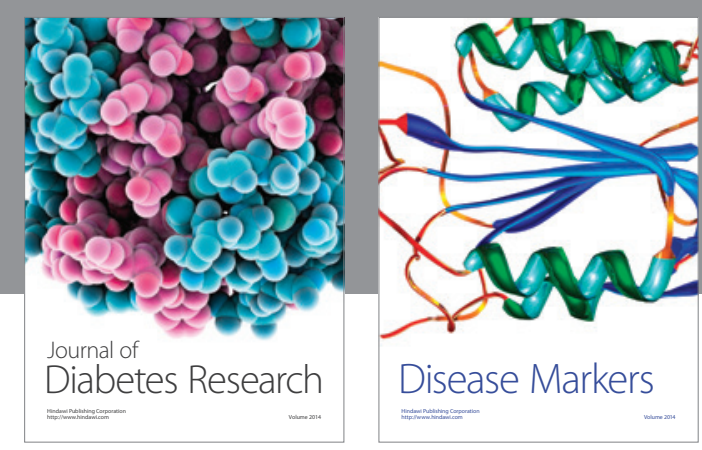

Disease Markers
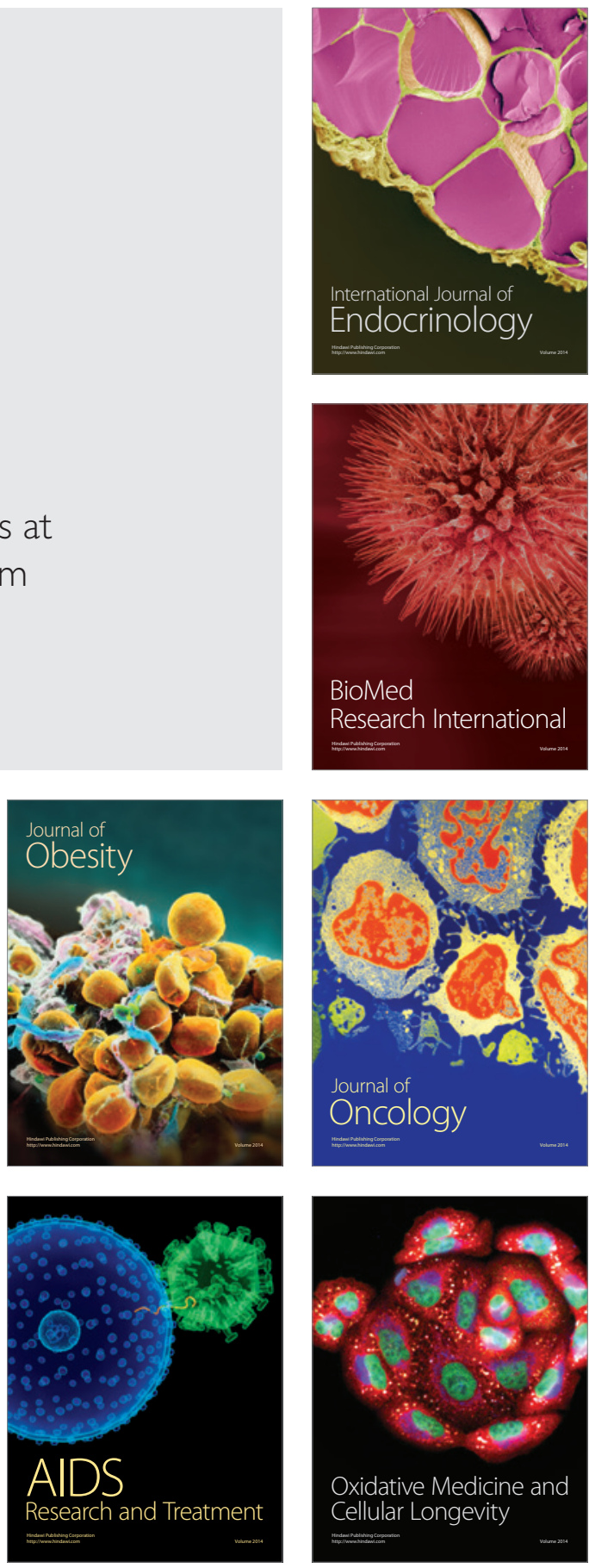